SLAC-PUB-13512

January 2009

\title{
Searches for Fractionally Charged Particles: What Should Be Done Next?
}

\author{
Martin L. Perl ${ }^{\mathrm{a}}$ \\ a SLAC National Accelerator Laboratory, Stanford University, \\ 2575 Sand Hill Road, Menlo Park, CA 94025, USA
}

Since the initial measurements of the electron charge a century ago, experimenters have faced the persistent question as to whether elementary particles exist that have charges that are fractional multiples of the electron charge. I concisely review the results of the last 50 years of searching for fractional charge particles with no confirmed positive results. I discuss the question of whether more searching is worthwhile?

\section{THE PUZZLE OF UNIT ELECTRIC CHARGE}

We have no explanation why the electric charges of all the known elementary particles are either zero or $q$ or $\pm \frac{1}{3} q$ or $\pm \frac{2}{3} q$ or $\pm q$ where $q$ is the magnitude of the electrons charge, $1.602 \times$ $10^{-19}$ coulombs. We call $q$ the unit electric charge.

There are no confirmed observations of elementary or composite particles with charge $Q=r q$ where $r$ is a fraction such as $\frac{2}{7}$ or an irrational or transcendental number. We call these hypothetical particles fractional electric charge particles, even though the fraction $\frac{Q}{q}$ might be greater than 1 ; for example a particle with charge $Q=\pi q$. We use $\mathrm{F}$ to mean a fractional electric charge particle.

My colleagues, Dinesh Loomba and Eric Lee, and I are publishing a detailed review of fractional charge searches [1]. Therefore, I give a few references in this paper and refer the reader to Ref. 1 for details and full references.

This paper will be published in the Proceedings of the $\mathrm{X}$ INTERNATIONAL WORKSHOP ON TAU LEPTON PHYSICS held at the Budker Institute of Nuclear Physics, Novosibirsk, Russia, September 22 - 25, 2008.

\section{QUARKS AND FREE QUARK SEARCHES}

About 1910 Robert Millikan and Harvey Fletcher elucidated the magnitude of the elec- tron charge $q[2]$. And by the early 1920s there was consensus that $q$ was the smallest electric charge. This was not challenged until the 1960s when physicists adopted the view of quarks as real elementary particles. This view of quarks and the increasing use of particle accelerators led to many searches for particles with charge $\frac{1}{3} q$ or $\frac{2}{3} q$ or higher fractions such as $\frac{4}{3} q$.

\section{SEARCH METHODS AND RE- MARKS ON SEARCHES}

There are five types of searches for fractional charge particles: searches using particle accelerators and fixed targets, searches using particle colliders, searches in cosmic rays, searches in bulk matter, and special search methods for particles with $Q$ very close to 0 called millicharged or minicharged particles.

We do not know how fractional charge particles interact with ordinary particles: is the interaction strong or electromagnetic or weak or a not yet discovered force?

Since we do not know the F mass, $m_{F}$, searches using accelerators, colliders, or cosmic rays are broader as the energy increases. On the other hand, the sensitivity of searches in bulk matter is independent of $m_{F}$.

Invited talk presented at the 10th International Workshop on Tau Lepton Physics (TAU08)
9/22/2008 - 9/25/2008, Novosibirsk, Russia

Work supported in part by DOE Contract No. DE-AC02-76SF00515. 


\section{ACCELERATOR AND COLLIDER SEARCHES}

General accelerator and collider detectors cannot be used to find particles with $\frac{Q}{q}<\frac{1}{3}$ because track reconstruction is uncertain. With this constraint no fractional charge particles have been found up to masses of about $200 \mathrm{GeV} / \mathrm{c}^{2}$ in searches using hadron collisions and up to about $100 \mathrm{GeV} / \mathrm{c}^{2}$ in searches using electron-positron collisions.

\section{SEARCHES FOR FRACTIONAL CHARGE PARTICLES COMING FROM OUTSIDE THE EARTH}

The possible sources are:

- The particles may have been produced in the early universe and still be a stable component of the present material in the universe.

- The particles may be produced in the present era in violent astrophysical processes .

- The particles may be produced in the interaction of ordinary cosmic rays with the Earths atmosphere.

No fractional charge particles have been found and search sensitivities have reached down to fluxes of less than $10^{-15} \mathrm{~cm}^{-2} \mathrm{~s}^{-1} \mathrm{sr}^{-1}$. Reference [3] describes the most sensitive search by the MICRO experiment.

\section{SEARCHES FOR FRACTIONAL CHARGE PARTICLES IN BULK MATTER}

Two methods have been used to search for fractional charge particles in bulk matter. The levitometer method was developed and used by the Smith and Jones group [4] and by Marinelli and Morpurgo [5].

The modern Millikan liquid drop method was pioneered by the Bland group at San Francisco State University [6]. The Millikan liquid drop method was then further developed and used at the SLAC National Accelerator Center from 1994 through 2007 with the initial paper by Charles Hendricks, Klaus Lackner, Gordon Shaw and myself [7]. Our final experimental paper, P. C. Kim et al. [8], reported null results on both meteoritic material and on the largest sample of bulk matter in the form of mineral oil ever studied.

The methods are both described in [1]. Searches have been carried out in the following materials: sea water, residues from evaporation of sea water, mercury, iron, niobium, silicone oil, mineral oil, and meteoritic material from asteroids.

There were no confirmed discoveries of fractional charged particles. Table 1 lists the major searches and [1] gives references and details.

\section{MILLICHARGED PARTICLES}

Millicharged particles are hypothetical elementary particles with small $\frac{Q}{q}$, usually $\frac{Q}{q}<0.1$, and extending down to $\frac{Q}{q}<10^{-8}$ or lower.

There have been many limitations on the existence of such particles from considerations such as energy loss in stars [9].

But there has been only one experimental search, by Prinz et al. [10]. No millicharged particles were found.

\section{WHAT SHOULD BE DONE NEXT?}

Thus the physics world has seen 50 years of failures in searches for fractional charge elementary particles. What should be done next?

First, some history of the searches that have been made by my colleagues and myself using the Millikan liquid drop method, searches from 1994 to 2007. Over the years we have been a creative, happy, and efficient band of experimenters consisting for various lengths of time of Gordon Shaw, Charles Hendricks, Klaus Lackner, Howard Rogers, Eric Lee, Nancy Mar, George Fleming, Brendan Casey, Edward, Garwin, Peter Kim, Sewan Fan, Irwin Lee, Valerie Halyo and myself. We ranged from undergraduate students to faculty. We had a wonderful time.

But what now? The funding for our group has changed and our group is dispersed; only Peter 
Table 1

Major searches in bulk matter for fractional charge elementary particles. All searches were null. In the table lev. means levitometer. The number of nucleons in the sample should be interpreted as the sensitivity of the search.

\begin{tabular}{llll}
\hline Method & Material & Sample $(\mathrm{mg})$ & Number of nucleons \\
\hline ferromagnetic lev. & steel & 3.7 & $2.2 \times 10^{21}$ \\
\hline ferromagnetic lev. & tungsten & 3.0 & $1.8 \times 10^{21}$ \\
\hline ferromagnetic lev. & niobium & 6.5 & $3.9 \times 10^{21}$ \\
\hline ferromagnetic lev. & meteorite & 2.8 & $1.7 \times 10^{21}$ \\
\hline liquid drop & sea water & 0.05 & $2.9 \times 10^{19}$ \\
\hline liquid drop & mercury & 2.0 & $1.2 \times 10^{21}$ \\
\hline liquid drop & silicone oil & 70.1 & $4.2 \times 10^{22}$ \\
\hline liquid drop & mineral oil & 259 & $1.6 \times 10^{23}$ \\
\hline liquid drop & meteorite & 3.9 & $2.3 \times 10^{21}$ \\
\hline
\end{tabular}

Kim, Howard Rogers and I remain at the SLAC National Accelerator Laboratory. Is it time to give up on fractional charge searches until the next generations of women and men in physics develop more insight into the theoretical questions involved in the fractional charge hypothesis? Is it time to give up on fractional charge searches until the next generations of women and men in physics invent a new, splendid and powerful search method for fractional charge particles? In thinking about this dilemma I have found Galisons book HOW EXPERIMENTS END [11] helpful.

Unless I get a splendid idea I will not continue experimental work in the fractional charge search field, but I do enjoy thinking about the field and dreaming about its possibilities. Meanwhile here are my recommendations:

1. New searches should increase sensitivities by at least a factor of 100 .

2. The MACRO experiment [3] was so extensive that it will be very expensive to improve upon itm for a new search in cosmic rays for fractional charge particles

3. The millicharged particle search of Prinz et al. [10] should be extended to higher energies and larger statistics

4. Searches for fractional charged particles at the Large Hadron Collider are difficult because most events have large multiplicity.
However the ingenuity of experimenters will find ways. Such searches should be done.

5. If searches in bulk matter are continued, the ferromagnetic levitometer method should be used. (Meteoritic material from asteroids is most appropriate for future examination.)

\section{ACKNOWLEDGMENTS}

I thank Simon Eydelman for inviting me to the TAU08 Workshop and giving me the opportunity to present this report even though it was not the subject of the Workshop. I thank my research colleagues of many years, Eric Lee and Dinesh Loomba who are the joint authors of [1], and who have taught me so much. I thank Garrett Morton for formatting and proofing this paper and putting it into LATEX. This work was supported by Contract DE-AC02-76SF00515 with the U. S. Department of Energy.

\section{REFERENCES}

1. M. L. Perl, E. R. Lee and D. Loomba, Searches for Fractionally Charged Particles, to be published in Ann. Rev. Nucl. Part. Science.

2. R. A. Millikan, A New Modification of the Cloud Method of Determining the Elementary Electrical Charge and the most Probable Value of that Charge, Philos. Mag., 19, 209 (1910). 
Millikan does not even acknowledge the vital contributions of his graduate student, Harvey Fletcher.

3. M. Ambrosio et al., Final Search for Lightly Ionizing Particles with the MACRO Detector, arXiv:hep/0402006v1, (2004).

4. P. F. Smith, SEARCHES FOR FRACTIONAL ELECTRIC CHARGE IN TERRESTRIAL MATTER, Ann. Rev. Nucl. Part. Science, 39, 73 (1989).

5. M. Marinelli and G. Morpurgo, The Electric Neutrality Of Matter: A Summary, Phys. Lett., B137, 439 (1984).

6. M. L. Savage et al., A SEARCH FOR FRACTIONAL CHARGE IN NATIVE MERCURY, Phys. Lett., 167B, 481 (1986).

7. C. D. Hendricks et al., Efficient bulk search for fractional charge with multiplexed Millikan chambers, Meas. Sci. Technol. 5, 337 (1994).

8. P. C. Kim et al., Search for Fractional-Charge Particles in Meteoritic Material, Phys. Rev. Lett., 99, 161804 (2007).

9. S. Davidson, S. Hannestad and G. Raffelt, Updated bounds on millicharged particles, JHEP 0005:003 (2000).

10. A. Prinz et al., Search for millicharged particles at SLAC, Phys. Rev. Lett., 81, 1175 (1998).

11. P. Galison, HOW EXPERIMENTS END, (Chicago, Univ. Chicago Press, 1987). 